\section{Tadalafil bei PAH zugelassen}

Mit Tadalafil hat die Europäische Kommission unter dem Handelsnamen Adcirca ${ }^{\circledR}$ einen weiteren Phosphodiesterase-(PDE-)5-Hemmer zur Behandlung von Patienten mit pulmonal-arterieller Hypertonie (PAH) zugelassen. Das seit Februar in Deutschland verfügbare neue Präparat muss nur einmal täglich in einer Dosis von $40 \mathrm{mg}$ eingenommen werden. Der Zulassung ging die Phase-IIIStudie PHIRST (Pulmonary Arterial Hypertension and Response to Tadalafil) voraus. Darin erziehlten die Patienten mit dem neuen Präparat im Vergleich zu Placebo bei guter Verträglichkeit signifikant bessere Ergebnisse im 6-MinutenGehtest sowie in der Zeit bis zum Eintreten einer klinischen Verschlechterung. PDE-5-Hemmer wirken über eine Dilatation der pulmonalen Blutgefäße.

Die lange Halbwertszeit von Tadalafil ( 16 Stunden) ermöglicht einen konstanten Wirkstoffspiegel mit nur einer Einnahme von $40 \mathrm{mg}$ täglich. Die aktuelle Zulassung umfasst PAH der WHO-Stadien II und III.

1. Galiè N et al. Circulation 2009;119(22):2894-903.

Lilly

\section{Fragen zum schnellen Erkennen unzureichender Asthmakontrolle}

Wenige gezielte Fragen helfen, die Asthmakontrolle schnell einzuschätzen und zu dokumentieren. Hierfür hat JanssenCilag einen Fragebogen zur Anwendung in der Praxis entwickelt. Der Inuvair ${ }^{\circledR}$ Symptomblock ist über den Außendienst des Unternehmens erhältlich. red

Janssen-Cilag

\section{Plan zur Terminvergabe bei SIT mit 5-Gräser-Tablette}

Speziell für Patienten, die mit der 5-Gräser-Tablette Oralair ${ }^{\circledR}$ behandelt werden, gibt es einen kostenlosen Terminplan. Wer auf der Pappscheibe den Zeiger auf den gewünschten Therapiebeginn setzt, erkennt u. a. auf einen Blick die Termine für die Folgeschritte. Bestellt werden kann der Plan über den Außendienst des Herstellerunternehmens oder unter der Telefonnummer $028429040-146$. red

Stallergenes

PNEUMONEWS

Herausgeber Klinik:

Prof. Dr. A. Gillissen, Leipzig

Priv.-Doz. Dr. M. J. Kohlhäufl, Stuttgart-

Gerlingen

\title{
Schriftleiter:
}

Priv.-Doz. Dr. S. Budweiser, Regensburg (Beatmungsmedizin)

Priv.-Doz. Dr. G. Friedel, Stuttgart-Gerlingen (Thoraxchirurgie)

Prof. Dr. A. Ghofrani, Gießen, Dr. K. Gutjahr, Leipzig (Erkrankungen des Lungenkreislaufs)

Prof. Dr. A. Gillissen, Leipzig (Asthma bronchiale)

Prof. Dr. M. Griese, München (Pädiatrische Pneumologie)

Prof. Dr. A. Günther, Priv.-Doz. Dr. Ph. Markart, Gießen (Interstitielle Lungenerkrankungen)

Priv.-Doz. Dr. M. J. Kohlhäufl, Stuttgart-Gerlingen (Tumoren)

Dr. S. R. Ott, Bern (Infektiologie)

Prof. Dr. K. Rasche, Wuppertal, Prof. Dr. K.-H. Rühle, Hagen, Prof. Dr. H. Schäfer, Völklingen (Schlafmedizin)

Prof. Dr. G. Rohde, Maastricht/Niederlande (COPD, Emphysem)

Dr. K. Schultz, Bad Reichenhall (Rehabilitation)

Prof. Dr. M. Schwab, Prof. Dr. K. Mörike, Tübingen (Pharmakologische Therapie)

\section{Redaktion:}

Dr. rer. nat. Beate Schumacher (Chefredaktion, verantwortlich, Anschrift wie Verlag, beate.schumacher@springer.com)

Ute Kempf (ute.kempf@springer.com), Dr. Judith Neumaier (judith.neumaier@t-online.de), Sarah Pampel (Ärztin), Angelika Holzgreve (Assistenz Chefredaktion, angelika.holzgreve@springer. com)

\section{Verlag:}

Springer Medizin, $\odot$ Urban \& Vogel GmbH, Aschauer Str. 30, D-81549 München,

Tel.: o 89/20 30 43-13 00, Fax: -13 99, http://www.urban-vogel.de

\section{Geschäftsführer:}

Harm van Maanen, Dr. med. Dirk Einecke, Ulrich Huber, Stephan Kröck, , Dr. med. Esther Wieland, Matthias Wissel.

\section{Anzeigenverkauf:}

Ines Spankau (Anzeigenleitung, -1339, verantwortlich, Anschrift wie Verlag), Anzeigenpreisliste Nr. 3 vom 1. Januar 2010

\section{Corporate Publishing:}

Dr. Ulrike Fortmüller (Leitung München, -1417)

\section{Herstellung:}

Frank Hartkopf (Leitung, -13 11); w\&Co MediaServices München GmbH \& Co KG (Layout); Dieter L. Adam, München (Titelbild)

\section{Vertrieb/Marketing Services:}

Frank Niemann (Leitung, -14 11)

\section{Abonnement:}

Die Zeitschrift erscheint 6 x jährlich. Neue Bezugspreise ab 2010: Einzelheft $12 €$ inkl. der deutschen MwSt. zuzügl. 2,50 € Versandkosten; Jahresabo $75 €$; Studenten/Ärzte in Aus- und Weiterbildung $60 €$, jeweils zuzügl. Versand (Inland 19,50 $€$, Ausland $35 €$ ).

Bestellungen nimmt der Verlag und jede Buchhandlung entgegen. Das Abonnement verlängert sich jeweils um ein Jahr, wenn es nicht zwei Monate vor Ende des Bezugszeitraums abbestellt wurde.

\section{Abonnentenservice:}

Tel.: 062 21/3 45-43 04; Fax: o 62 21/3 45-42 29

Gesamtherstellung: Stürtz $\mathrm{GmbH}$, AlfredNobel-Str. 33, D-97080 Würzburg

Originalien: Zur Veröffentlichung kommen nur Arbeiten, die an anderer Stelle weder angeboten noch erschienen sind. Die Autoren sind verpflichtet zu prüfen, ob Urheberrechte Dritter berührt werden. Alle CME-Manuskripte werden von unabhängigen Experten begutachtet. Eine redaktionelle Bearbeitung bleibt vorbehalten. Autoren, die mit vollem Namen genannt sind, veröffentlichen ihre Beiträge in alleiniger Verantwortung.

Copyright: Der Verlag behält sich das ausschließliche Recht der Verbreitung, Übersetzung und jeglicher Wiedergabe auch von Teilen dieser Zeitschrift durch Nachdruck, Fotokopie, Mikrofilm, EDV-Verwertung, Funk- oder Fernsehaufzeichnung vor. Jede gewerblich hergestellte oder benutzte Fotokopie verpflichtet nach $§ 54$ (2) UrHG zur Gebührenzahlung an die VG Wort, Abt. Wissenschaft, Goethestr. 49, D-80336 München, von der die Modalitäten zu erfragen sind.

Rechtseinräumung der Autoren: Mit der Einsendung eines Manuskripts zur Veröffentlichung überträgt der Verfasser dem Verlag für den Fall der Annahme das Recht, das Manuskript geändert oder unverändert, ganz oder teilweise in Pneumo-News und anderen Publikationen der Fachverlagsgruppe, in den zugehörigen Onlinediensten, in Onlinedatenbanken Dritter und, soweit vereinbart, gegen Nachhonorar in Sonderdrucken für Industriekunden zu nutzen.

Hinweis: Die in dieser Zeitschrift angegebenen Dosierungen - vor allem von Neuzulassungen - sollten in jedem Fall mit dem Beipackzettel der verwendeten Medikamente verglichen werden.

ISSN 1865-5467

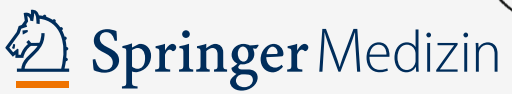

